ARTICLE

Received 11 Apr 2015 | Accepted 17 Sep 2015 | Published 23 Oct 2015

DOI: $10.1038 /$ ncomms9655 OPEN

\title{
Single-photon non-linear optics with a quantum dot in a waveguide
}

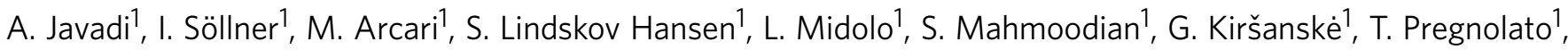 \\ E.H. Lee ${ }^{2}$, J.D. Song ${ }^{2}$, S. Stobbe ${ }^{1} \&$ P. Lodahl ${ }^{1}$
}

Strong non-linear interactions between photons enable logic operations for both classical and quantum-information technology. Unfortunately, non-linear interactions are usually feeble and therefore all-optical logic gates tend to be inefficient. A quantum emitter deterministically coupled to a propagating mode fundamentally changes the situation, since each photon inevitably interacts with the emitter, and highly correlated many-photon states may be created. Here we show that a single quantum dot in a photonic-crystal waveguide can be used as a giant non-linearity sensitive at the single-photon level. The non-linear response is revealed from the intensity and quantum statistics of the scattered photons, and contains contributions from an entangled photon-photon bound state. The quantum non-linearity will find immediate applications for deterministic Bell-state measurements and single-photon transistors and paves the way to scalable waveguide-based photonic quantum-computing architectures.

\footnotetext{
${ }^{1}$ Niels Bohr Institute, University of Copenhagen, Blegdamsvej 17, DK-2100 Copenhagen, Denmark. ${ }^{2}$ Center for Opto-Electronic Convergence Systems, Korea Institute of Science and Technology, Seoul 136-791, Korea. Correspondence and requests for materials should be addressed to A.J. (email: Javadi@nbi.ku.dk) or to P.L. (email: Lodahl@nbi.ku.dk)
} 
T he access to an efficient optical non-linearity enables the processing of quantum information stored in light and generation of exotic states of light ${ }^{1-5}$. A non-linearity capable of operating down to the ultimate level of single photons has been long sought after, as it opens new avenues for photonic quantum-information architectures ${ }^{6,7}$, and enables efficient Bell-state analysers ${ }^{8}$ and single-photon transistors ${ }^{9}$. Moreover, such an efficient non-linearity could have extensive applications in optical signal processing ${ }^{10}$ and improve linear-optics quantum-information architectures ${ }^{11}$. Photonic nanostructures provide a route to overcoming these limitations since light and matter can be deterministically interfaced. One approach to photon non-linearities exploits the anharmonic spectrum of a cavity polariton, which has been experimentally demonstrated both with atoms ${ }^{12}$ and quantum dots ${ }^{13-17}$. An alternative approach uses the intrinsic non-linearity of a quantum emitter deterministically coupled to a single photonic mode (a 'one-dimensional (1D)atom'); such a coupling was recently achieved with single quantum dots in photonic-crystal waveguides ${ }^{18}$. Theoretical studies have predicted that intricate photon-photon ${ }^{3}$ and photon-emitter ${ }^{19}$ entanglement (so-called bound states) may be induced by deterministic few-photon scattering. So far a quantum-emitter non-linearity has been observed at microwave frequencies with superconducting qubits $^{20}$, at optical frequencies with single atoms in cavities ${ }^{21-23}$ and with single molecules in dielectric nanoguides ${ }^{24}$.
A quantum dot in a photonic waveguide is a particularly attractive approach to quantum non-linear optics since it can be naturally incorporated in integrated photonic circuits. For quantum dots in photonic cavities ${ }^{14,15}$ the precise tuning of the emitter to the cavity resonance is required. Moreover, the efficient outcoupling of photons from a high-Q photonic-crystal cavity can be challenging due to the complex mode structure of nanophotonic cavities, and coupling of $\mathrm{PhC}$ cavities to in-plane propagating modes compromises the Q-factor of the cavity. The very wide coupling bandwidth in photonic-crystal waveguides ${ }^{25}$ implies that scalable quantum architectures may be envisioned with significantly less experimental overhead, since only the quantum dot needs to be tuned. Also the planar propagation of the light in a waveguide enables a quantum-photonic chip, where all the required operations can be done on the same chip.

A quantum dot in a photonic-crystal waveguide constitutes the paradigmatic example of a $1 \mathrm{D}$ artificial atom where light-matter interaction is fundamentally different from that in $3 \mathrm{D}$. For instance, dipole-induced single-photon interference may be studied, which can be considered a precursor to the experimental demonstration of single-photon non-linearity and photonphoton bound states. Furthermore, an anomalous radiative Lamb shift or infinitely ranging dipole-dipole interaction have been been predicted ${ }^{5}$.

The present work reports on single-photon non-linear optics in a waveguide-based scalable solid-state platform. We design a a

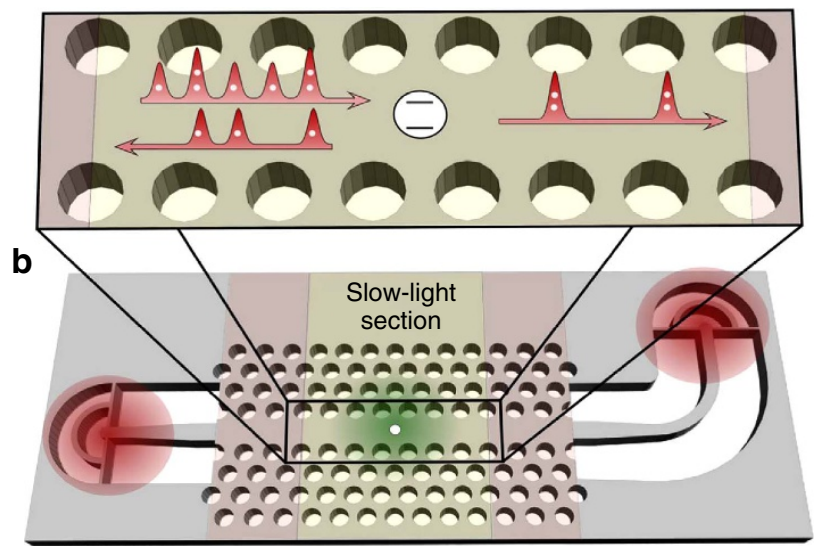

C

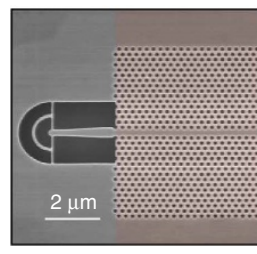

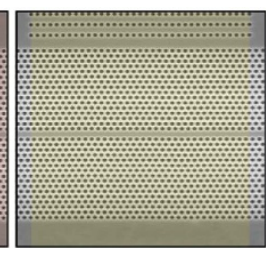

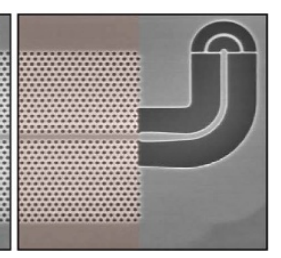

d

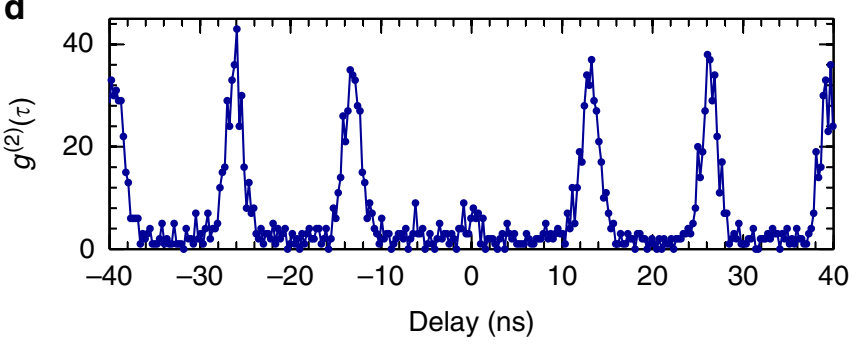

e

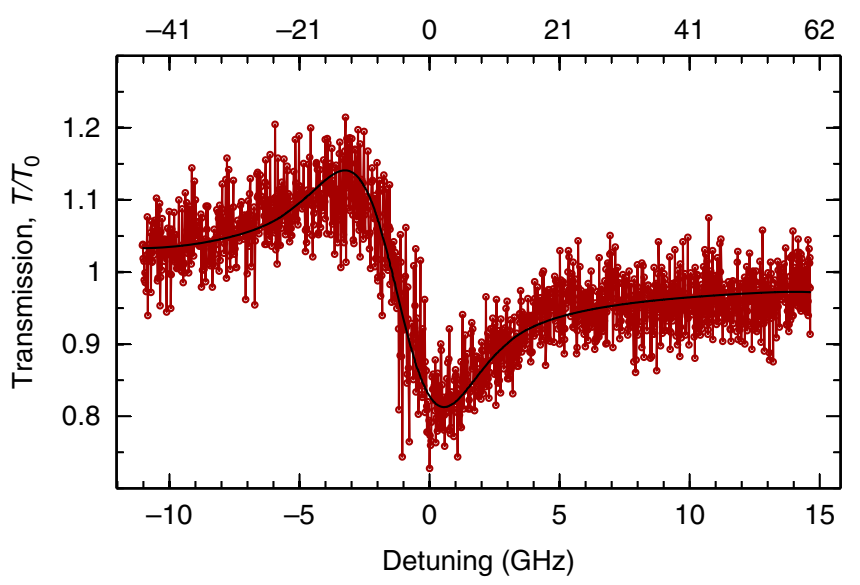

Figure 1 | Resonant spectroscopy on a quantum dot in a photonic-crystal waveguide. (a) Operational principle of resonant scattering in a photoniccrystal waveguide. Single-photon components of the incoming light are reflected by the quantum dot while two- and more-photon components are preferentially transmitted. (b) Illustration and (c) scanning electron micrograph of the sample. A quantum dot (white circle) in the central part of the slow-light section is excited by launching light through one grating and detecting light from the other grating. The red areas indicate the size of the excitation and collection areas. The green area is the illumination region of the repump laser, see Supplementarty Information. (d) Autocorrelation function of the photons emitted from the target quantum dot. The central peak is highly suppressed, which proves the single-photon character of the system. The area under the suppressed peak is about $30 \%$ of the adjacent peaks. (e) Resonant transmission spectrum recorded by scanning a narrow-band continuous-wave laser through the resonance of a quantum dot in the case of weak excitation. The power on the sample was $50 \mathrm{pW}$, which is far below the critical power of $1.9 \mathrm{nW}$. The solid black line is a model of the experimental data of the Fano resonance, see Supplementarty Information for details of the model. 
a

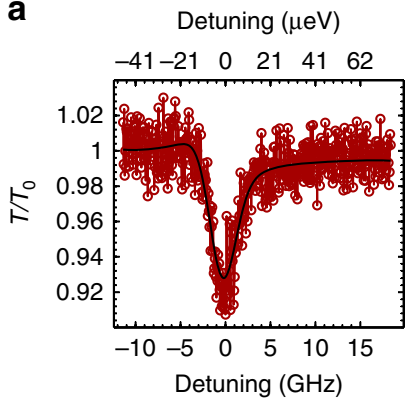

b

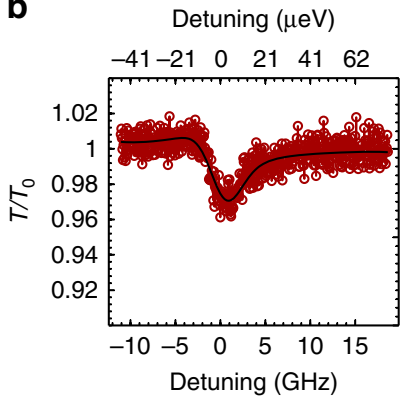

C

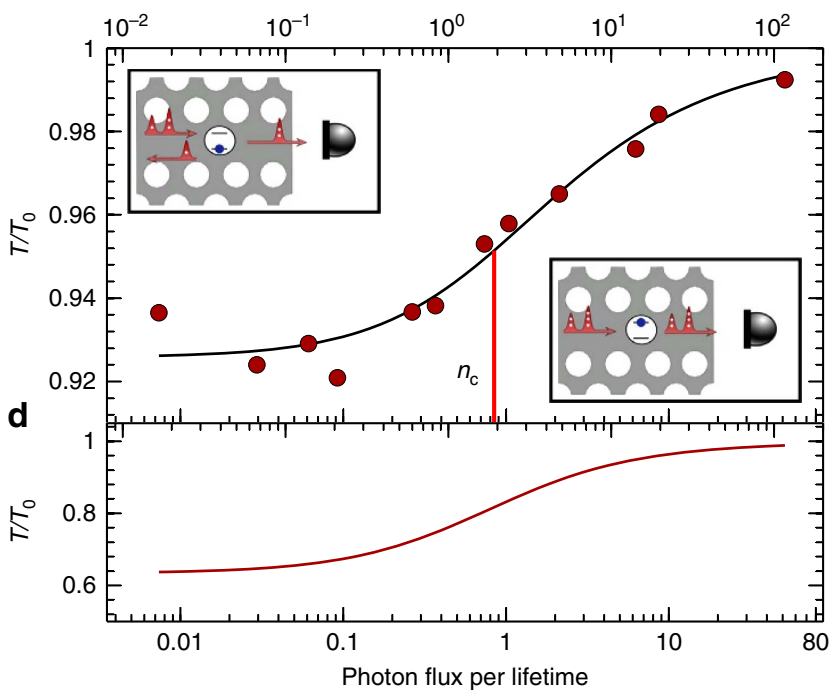

Figure 2 | Non-linear response of a single quantum dot in a photoniccrystal waveguide. Examples of transmission spectra recorded at two different powers of (a) $P=0.18 \mathrm{nW}$ and (b) $P=2.2 \mathrm{nW}$. (c) Transmission on resonance with the quantum dot versus incident photon flux relative to the emitter lifetime. The top axis shows the corresponding optical power applied to the sample. The solid line is a fit to the experimental data. The critical power that characterizes the saturation curve is indicated on the axis. The insets show the measurement geometry and illustrate that for weak excitation the quantum dot preferentially reflects while it becomes transparent at stronger excitation where two- and higher-photon components of the coherent state dominate. (d) Same as (c) but after deconvolution of the spectral diffusion and blinking.

photonic circuit that allows us to probe single quantum dots, efficiently coupled to photonic-crystal waveguides. We show that a single quantum dot can modify the transmission of the waveguide by up to $30 \%$. Our experimental results show that the transmission is highly non-linear and the non-linearity operates at the single-photon level. The critical average number of photons per lifetime of the emitter is 0.81 . We also show that the statistics of the transmitted photons is bunched, which reveals the quantum nature of the non-linearity.

\section{Results}

Operational principle and sample structure. The operational principle of the quantum-dot non-linearity is outlined in Fig. 1a: by scattering a weak resonant laser on a single quantum dot in the photonic-crystal waveguide, the single-photon component is reflected while two- and higher-photon components have an increased probability of being transmitted. The layout and scanning electron micrograph of the sample are shown in
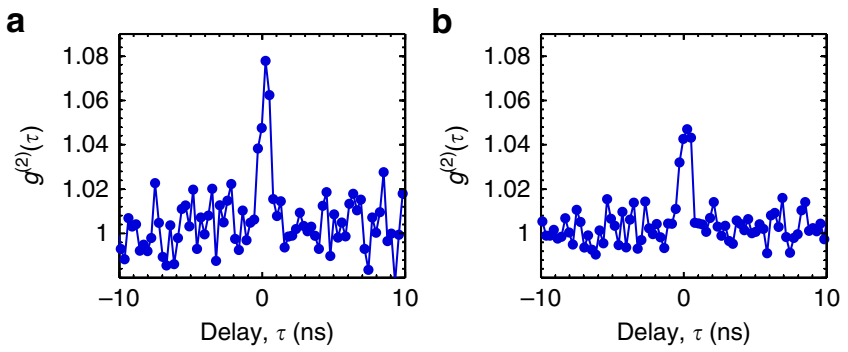

C

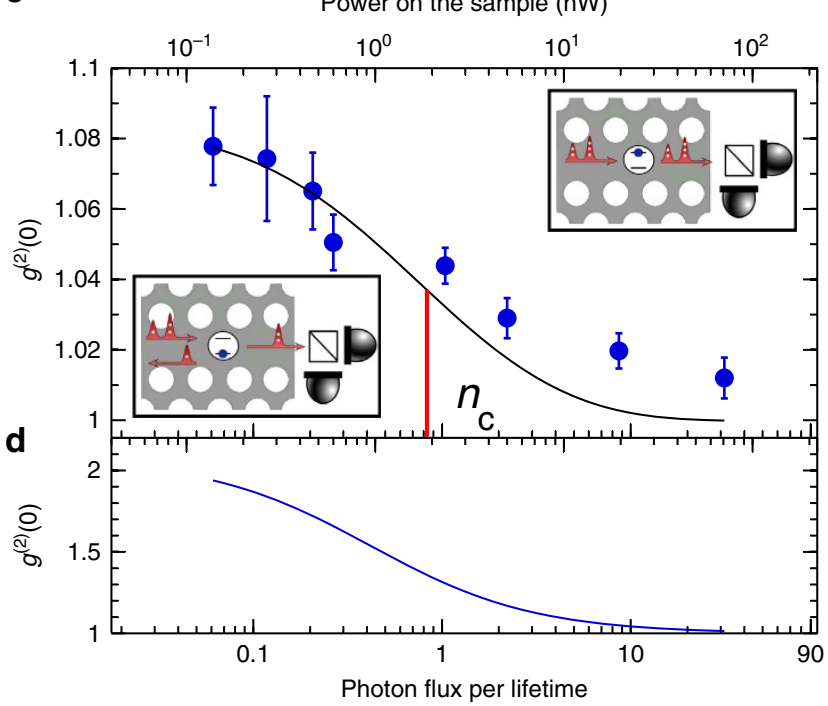

Figure 3 | Photon statistics induced by the single-photon non-linearity.

$(\mathbf{a}, \mathbf{b})$ Measurements of the autocorrelation function of the transmitted light recorded on resonance with the quantum dot for the conditions corresponding to Fig. 2a,b, respectively. (c) Power dependence of the autocorrelation function peak at $\tau=0$. The solid black line is a fit to the data. A maximum bunching of $8 \%$ is observed, which corresponds to $18 \%$ when accounting for the finite response time of the detection. The vertical line indicates the critical power. (d) Same as (c) but after deconvolution of the spectral diffusion and blinking.

Fig. 1b,c, respectively. The sample consists of a central slow-light waveguide section (slow-down factor $n_{\mathrm{g}} \sim 30$ ) terminated on each side by two waveguide sections $\left(n_{\mathrm{g}}=5\right)$ and coupled through suspended waveguides to two gratings, which direct the emission vertically out of the planar structure. One grating is used for launching light into the waveguide and the other for extracting the transmitted light. Furthermore, the quantum dot is exposed to a repump laser to prepare and stabilize the initial state of the emitter before the scattering process. The detailed description of the sample and experimental procedure is presented in Supplementary Note 1 and 2, and Supplementary Figs 1 and 2. We choose a quantum dot line that has a wavelength of $\sim 914 \mathrm{~nm}$ for this experiment, see Supplementary Note 3 and Supplementary Fig. 3 for the emission spectrum of the sample. We verify that a single quantum dot is probed by recording the autocorrelation function of the emitted photons under pulsed excitation. Figure 1d shows the autocorrelation function versus time delay, while exciting close to the saturation power of the quantum dot. The antibunching of the photons observed around zero time delay proves the single-photon nature of the emission, see the Supplementary Note 4 and Supplementary Fig. 4 for the details of the measurement and the analysis. Figure le shows an example of the transmission spectrum recorded when scanning a narrow-linewidth laser through the resonance feature of a 
quantum dot coupled to a photonic-crystal waveguide. These measurements are performed at low excitation powers in the coherent-scattering regime where the incoming and outgoing fields maintain a fixed phase relation. Residual reflections from the waveguide ends imply that weak Fabry-Perot resonances form, which lead to a characteristic Fano line shape $e^{26,27}$ of $\sim 30 \%$ modulation.

The peak (dip) of the transmission spectrum corresponds to dipole-induced transparency (reflection) resulting from singlephoton interference. The size of the modulation is determined by the $\beta$-factor, which quantifies the coupling efficiency, as well as the spectral diffusion and blinking of the quantum-dot line due to charge or spin noise $\mathrm{e}^{28}$, and residual broadening of the zero-phonon line, for example, due to carrier-phonon interactions ${ }^{29}$. Spectral diffusion and blinking processes can be strongly reduced by implementing electrical gates on the quantum-dot samples ${ }^{28}$ and can be further suppressed through active stabilization.

Non-linear transmission of the waveguide. The non-linear response of the quantum dot is investigated by recording the transmission as a function of excitation power. Two examples of transmission spectra are displayed in Fig. 2a,b for weak and intermediate powers, respectively. In this data set a transmission dip of $\sim 8 \%$ was recorded, limited by spectral diffusion, which was found to vary when the sample was heated up and subsequently cooled down. Figure $2 \mathrm{c}$ shows the transmission as a function of power inside the waveguide and displays a characteristic non-linear saturation behaviour. The data are modelled very well by the theory of ref. 30 ( $c f$. Supplementary Notes 5-7 and Supplementary Figs 5 and 6 for a detailed account) for a coupling efficiency of $\beta=85 \%$, broadening by spectral diffusion of $\sigma / \Gamma=3.6$, blinking probability of $\alpha=0.43$, and a pure dephasing rate describing the broadening of the zero-phonon line of $\gamma_{0} / \Gamma=0.79$. Here the emitter decay rate $\Gamma=2.5 \mathrm{~ns}^{-1}$ is obtained in time-resolved measurements leading to an independent measurement of the coupling efficiency of $\beta \sim 96 \%$ (ref. 18). The two values are consistent since the $\beta$-factor extracted from resonant scattering experiments is effectively reduced compared with the value obtained from the dynamics by the presence of weak phonon sidebands due to incoherent Raman scattering processes $^{31}$. The incoherently scattered photons are not distinguishable from the coherently scattered photons in timeresolved measurements, while they contribute differently in the present transmission experiment. To maximize the contrast of the signal, the intensity of the repump laser is varied between $250 \mathrm{pW}$ and $1.2 \mathrm{nW}$ for these measurements, see Supplementary Note 8 and Supplementary Fig. 7. Figure $2 \mathrm{~d}$ shows the non-linear response after deconvolution of the spectral diffusion and blinking, which can be suppressed by implementing electrical gates. We extract a critical photon flux per lifetime of $n_{\mathrm{c}}=0.81$ characterizing the non-linear saturation curve, demonstrating that the non-linearity operates at the ultimate level of single photons. This corresponds to a characteristic switching energy of only $\sim 0.17$ attojoule. For comparison, the state-of-the-art switching power in the classical optics is around several femtojoules per switching event ${ }^{32,33}$ and the observed nonlinearity has a big potential for very low-power optical switching. The input power applied to the sample at the critical power is $1.9 \mathrm{nW}$, which implies that $23 \%$ of the excitation beam is successfully coupled to the quantum dot in the waveguide. Photonic waveguide non-linearities are particularly promising for obtaining non-linearities at low photon numbers; a detailed comparison to the case of cavity polaritons is presented in Supplementary Note 9 and Supplementary Fig. 8.
Quantum nature of the non-linearity. By monitoring the photon statistics of the transmitted light, the quantum character of the nonlinear response and the ability to generate photon-photon bound states are revealed. In the ideal case of $\beta \rightarrow 1$ and for a weak coherent state, the single-photon component is fully reflected while two- and higher-photon components are preferentially transmitted leading to photon bunching in the transmission ${ }^{1,9}$. Figure $3 \mathrm{a}, \mathrm{b}$ show the experimental signature of photon bunching in the intensity autocorrelation function $g^{2}(\tau)$ of the transmission as a function of the delay $\tau$. The peak centred at $\tau=0$ is the experimental signature that two or more photons impinging on the quantum dot within its radiative lifetime interact leading to photon-photon bound states. We estimate a photon-photon bound-state contribution to the transmission probability of the two-photon component of $\sim 70 \%$, which is the entangled part of the scattered light (see Supplementary Note 10 and Supplementary Fig. 9 for further details). The observed photon bunching is highly sensitive to decoherence processes ${ }^{5}$ and the experimental observation of this effect testifies that resonant photon scattering using quantum dots is highly coherent ${ }^{34}$. The amount of photon bunching is found to decrease with increasing power ( $c f$. Fig. $3 c$ ) since two- and higher-photon components increasingly dominate the input state. At high excitation power, we observe $g^{2}(0) \approx 1$, corresponding to the Poissonian statistics of a coherent state, that is, the recorded light is unaffected by the saturated quantum dot. This experiment demonstrates the basic operational principle behind photon sorting with applications in photonic quantum-information processing ${ }^{8}$. Several control experiments were conducted to confirm that the observed photon bunching is mediated by the quantum dot. For example, bunching of the photons vanishes when the laser is detuned far from the resonance of the quantum dot. We also verified that $g^{2}(\tau)$ is a flat line when the repump laser is turned off, see Supplementary Note 11 and Supplementary Fig. 10 for more details. The observed degree of bunching is explained by three factors: pure dephasing and spectral diffusion of the quantum dot transition, as well as the limited instrument response function of the setup. After deconvolution of the latter, we obtain $g^{(2)}(0)=1.18$. We emphasize that the two independent set of experimental data of Figs $2 c$ and $3 c$ are well explained by theory for the same set of parameters. The inherent potential of the system is illustrated in Fig. 3d, which shows the expected amount of photon bunching after deconvoluting the slow decoherence processes found in the experiment. For the parameters of the present experiment and in the weak excitation limit, we predict $g^{(2)}(0) \sim 2.1$, which is mainly limited by the pure dephasing rate $\gamma_{0}$. Importantly, such decoherence has been shown to give a minor contribution in single-photon indistinguishability measurements on quantum dots controlled by electrical gates ${ }^{34}$, that is, even more dramatic photonphoton scattering processes should be obtainable.

\section{Discussions}

Having access to single-photon non-linearities may open new perspectives for processing both classical and quantuminformation encoded in photons. In the classical regime, it enables constructing ultimately energy-efficient optical switches that are triggered by just a few quanta of light, which is required to outperform electronic transistors ${ }^{33}$. In the quantum regime, it may enable new and resource-efficient functionalities required for deterministic quantum-information processing with photons ${ }^{35}$. With spectral and spatial control of the quantum dots ${ }^{36}$ the present system can be scaled to obtain multiple quantum dots deterministically coupled to a photonic-crystal waveguide, each inducing a giant non-linearity. Such a complex quantum system may be exploited for advanced quantum simulations and to engineer novel quantum states of coupled light and matter ${ }^{37}$. 


\section{References}

1. Rice, P. R. \& Carmichael., H. J. Single-atom cavity-enhanced absorption. I: photon statistics in the bad-cavity limit. IEEE J. Quantum Electron. 24, 1351-1366 (1988).

2. Kojima, K., Hofmann, H. F., Takeuchi, S. \& Sasaki., K. Nonlinear interaction of two photons with a one-dimensional atom: spatiotemporal quantum coherence in the emitted field. Phys. Rev. A 68, 013803 (2003).

3. Shen, J.-T. \& Fan., S. Strongly correlated two-photon transport in a one-dimensional waveguide coupled to a two-level system. Phys. Rev. Lett. 98, 153003 (2007)

4. Chang, D. E., Vuletić, V. \& Lukin., M. D. Quantum nonlinear optics-photon by photon. Nat. Photon. 8, 685-694 (2014).

5. Lodahl, P., Mahmoodian, S. \& Stobbe, S. Interfacing single photons and single quantum dots with photonic nanostructures. Rev. Mod. Phys. 87, 347-400 (2015).

6. Zheng, H., Gauthier, J. \& Baranger., H. U. Waveguide-QED-based photonic computation. Phys. Rev. Lett. 111, 090502 (2013).

7. Duan, L. M. \& Kimble., H. J. Scalable photonic quantum computation through cavity-assisted interactions. Phys. Rev. Lett. 92, 127902 (2004).

8. Witthaut, D., Lukin, M. D. \& Sørensen, A. S. Photon sorters and QND detectors using single photon emitters. Europhys. Lett. 97, 50007 (2012).

9. Chang, D. E., Sørensen, A. S., Demler, E. A. \& Lukin., M. D. A single-photon transistor using nanoscale surface plasmons. Nat. Phys. 3, 807-812 (2007).

10. Gibbs, H. Optical Bistability: Controlling Light With Light (Academic Press, 1985).

11. Knill, E., Laflamme, R. \& Milburn, G. J. A scheme for efficient quantum computation with linear optics. Nature 409, 46-52 (2001).

12. Birnbaum, K. M. et al. Photon blockade in an optical cavity with one trapped atom. Nature 436, 87-90 (2005).

13. Srinivasan, K. \& Painter, O. Linear and nonlinear optical spectroscopy of a strongly coupled microdisk-quantum dot system. Nature 450, 862-865 (2007).

14. Faraon, A. et al. Coherent generation of non-classical light on a chip via photon-induced tunnelling and blockade. Nat. Phys. 4, 859-863 (2008).

15. Reinhard, A. et al. Strongly correlated photons on a chip. Nat. Photon. 6, 93-96 (2012).

16. Loo, V. et al. Optical nonlinearity for few-photon pulses on a quantum dot-pillar cavity device. Phys. Rev. Lett. 109, 166806 (2012).

17. Kim, H., Bose, R., Shen, T. C., Solomon, G. S. \& Waks, E. A quantum logic gate between a solid-state quantum bit and a photon. Nat. Photon. 7, 373-377 (2013).

18. Arcari, M. et al. Near-unity coupling efficiency of a quantum emitter to a photonic crystal waveguide. Phys. Rev. Lett. 113, 093603 (2014)

19. Longo, P., Schmitteckert, P. \& Busch, K. Few-photon transport in lowdimensional systems: interaction-induced radiation trapping. Phys. Rev. Lett. 104, 023602 (2010).

20. Hoi, I.-C. et al. Generation of nonclassical microwave states using an artificial atom in 1D open space. Phys. Rev. Lett. 108, 263601 (2012).

21. Tiecke, T. et al. Nanophotonic quantum phase switch with a single atom. Nature 508, 241-244 (2014)

22. Reiserer, A., Kalb, N., Rempe, G. \& Ritter, S. A quantum gate between a flying optical photon and a single trapped atom. Nature 508, 237-240 (2014).

23. Volz, J., Scheucher, M., Junge, C. \& Rauschenbeutel, A. Nonlinear [pi] phase shift for single fibre-guided photons interacting with a single resonatorenhanced atom. Nat. Photon. 8, 965-970 (2014).

24. Faez, S., Türschmann, P., Haakh, H. R., Götzinger, S. \& Sandoghdar., V. Coherent interaction of light and single molecules in a dielectric nanoguide. Phys. Rev. Lett. 113, 213601 (2014).

25. Lund-Hansen, T. et al. Experimental realization of highly efficient broadband coupling of single quantum dots to a photonic crystal waveguide. Phys. Rev. Lett. 101, 113903 (2008).
26. Shen, J. T. \& Fan, S. Coherent photon transport from spontaneous emission in one-dimensional waveguides. Opt. Lett. 30, 2001-2003 (2005).

27. Goban, A. et al. Atom-light interactions in photonic crystals. Nat. Commun. 5, 3808 (2014).

28. Kuhlmann, A. V. et al. Charge noise and spin noise in a semiconductor quantum device. Nat. Phys. 9, 570-575 (2013).

29. Muljarov, E. \& Zimmermann., R. Dephasing in quantum dots: quadratic coupling to acoustic phonons. Phys. Rev. Lett. 93, 237401 (2004).

30. Auffèves-Garnier, A., Simon, C., Gérard, J.-M. \& Poizat, J.-P. Giant optical nonlinearity induced by a single two-level system interacting with a cavity in the Purcell regime. Phys. Rev. A 75, 053823 (2007).

31. Konthasinghe, K. et al. Coherent versus incoherent light scattering from a quantum dot. Phys. Rev. B 85, 235315 (2012).

32. Nozaki, K. et al. Sub-femtojoule all-optical switching using a photonic-crystal nanocavity. Nat. Photon. 4, 477-483 (2010).

33. Miller, D. A. Are optical transistors the logical next step? Nat. Photon. 4, 3-5 (2010).

34. Matthiesen, C. et al. Phase-locked indistinguishable photons with synthesized waveforms from a solid-state source. Nat. Commun. 4, 1600 (2013).

35. Ralph, T. C., Söllner, I., Mahmoodian, S., White, A. G. \& Lodahl, P. Photon sorting, efficient Bell measurements, and a deterministic controlled- $Z$ gate using a passive two-level nonlinearity. Phys. Rev. Lett. 114, 173603 (2015).

36. Jamil, A. et al. On-chip generation and guiding of quantum light from a site-controlled quantum dot. Appl. Phys. Lett. 104, 101108 (2014).

37. Chang, D. et al. Crystallization of strongly interacting photons in a nonlinear optical fibre. Nat. Phys. 4, 884-889 (2008).

\section{Acknowledgements}

We gratefully acknowledge financial support from the Villum Foundation, the Carlsberg Foundation, the Lundbeck foundation, the Danish Council for Independent Research (Natural Sciences and Technology and Production Sciences) and the European Research Council (ERC Consolidator Grant ALLQUANTUM). J.D.S. and E.H.L. acknowledge the support from the GRL and KIST institutional programs.

\section{Author contributions}

A.J., I.S. and S.M. designed the sample. A.J., I. S., M.A. and S.L.H. carried out the experiment and analysed the data with input from S. M. L.M., G.K. and T.P. fabricated the samples, E.H.L. and J.D.S. grew the quantum-dot material. P.L. and S.S. supervised the project. P.L., A.J., I.S. and S.M. prepared the manuscript with input from all authors

\section{Additional information}

Supplementary Information accompanies this paper at http://www.nature.com/ naturecommunications

Competing financial interests: The authors declare no competing financial interests.

Reprints and permission information is available online at http://npg.nature.com/ reprintsandpermissions/

How to cite this article: Javadi, A. et al. Single-photon non-linear optics with a quantum dot in a waveguide. Nat. Commun. 6:8655 doi: 10.1038/ncomms9655 (2015).

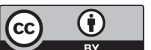

This work is licensed under a Creative Commons Attribution 4.0 International License. The images or other third party material in this article are included in the article's Creative Commons license, unless indicated otherwise in the credit line; if the material is not included under the Creative Commons license, users will need to obtain permission from the license holder to reproduce the material. To view a copy of this license, visit http://creativecommons.org/licenses/by/4.0/ 